\title{
Anterior Segment Biometry During Accommodation and Effects of Cycloplegics by Swept-Source Optical Coherence Tomography
}

This article was published in the following Dove Press journal: Clinical Ophthalmology

\section{Tadahiro Mitsukawa \\ Yumi Suzuki \\ Yosuke Momota \\ Shun Suzuki \\ Masakazu Yamada (1)}

Department of Ophthalmology, Kyorin University School of Medicine, Mitaka,

Tokyo I8I-86II, Japan
Correspondence: Masakazu Yamada Email yamadamasakazu@ks.kyorin-u.ac.jp
Purpose: We analyzed changes in the crystalline lens during accommodation and the effects of cycloplegics by swept-source anterior-segment optical coherence tomography (AS-OCT). Materials and Methods: Twenty healthy volunteers ( 7 males and 13 females, aged 22-34 years), with no history of eye disease except for refractive errors, were recruited. Biometric parameters, including anterior chamber depth (ACD), lens thickness (LT), and anterior and posterior curvature of the lens (ACL and PCL), were measured using AS-OCT (CASIA2). The measurements were performed with or without an accommodative demand of 5.0 diopters (D). The same tests were repeated following the topical administration of $1 \%$ cyclopentolate or a compounding agent comprising $0.5 \%$ tropicamide and $0.5 \%$ phenylephrine.

Results: The AS-OCT system was capable of simultaneous visualization of all optical components of the anterior segment in a single frame. ACD, LAC, and LPC decreased and LT increased significantly during 5.0 D accommodative stimulation in both eyes. Both cyclopentolate and tropicamide/phenylephrine eyedrops led to deeper ACD, thinner LT, and flatter LAC. There were no significant differences in all lens parameters despite having 5.0 D accommodative stimulation in both eyes with cycloplegia.

Conclusion: Our results suggest that both tropicamide/phenylephrine and cyclopentolate eyedrops have enough cycloplegic effects in young adults.

Keywords: accommodation, cycloplegics, lens, optical coherence tomography, refraction

\section{Introduction}

Refraction and accommodation are key elements of vision. ${ }^{1,2}$ Presbyopia and accommodative insufficiency are common causes of asthenopia. The precise measurements of refraction as well as the amplitude of accommodation are essential when glasses are prescribed. In some clinical situations, such as accommodative esotropia, accommodative spasm, and preoperative evaluation of refractive surgery, the exclusion of residual accommodation is crucial to evaluate the refractive state. ${ }^{3,4}$ Cycloplegic drugs that relax the ciliary muscle are commonly used to determine cycloplegic refraction.

Atropine is the gold standard for evaluating cycloplegic refraction. ${ }^{5,6}$ However, its long action, which results in prolonged blurred vision and a lengthy recovery time, has limited its application, particularly among elder children and adults. Besides atropine, the most commonly used cycloplegic agents are tropicamide and cyclopentolate. ${ }^{4}$ The major differences between these agents are duration of the onset, maximum efficacy, and recovery time. Cycloplegics effects on accommodation have been evaluated by 
measuring the refractive changes in optical power of the eye with an auto refractometer or a retinoscope. Several studies have been conducted to compare the cycloplegic effects of these agents, and different results have been reported. ${ }^{7-10}$ In addition, cross-sectional imaging methods, including magnetic resonance imaging and Scheimpflug camera imaging, are used to determine the mechanisms underlying accommodation and cycloplegic action. ${ }^{11-14}$

Anterior-segment optical coherence tomography (ASOCT) has evolved rapidly for noninvasive imaging of the anterior segment of the eye. ${ }^{15,16}$ In recent years, AS-OCT has been used to study the behavior of the ocular lens during accommodation in vivo. ${ }^{17-22}$ However, the simultaneous imaging of the entire anterior segment from the cornea through the crystalline lens is a challenge for ASOCT. Custom-made OCT systems have been used in most studies, because commercial AS-OCT instruments do not have enough depth range to capture the posterior surface of the lens. ${ }^{17-21}$ Recently, a newly developed swept-source AS-OCT system (CASIA2; Tomey Corp., Nagoya, Japan) has become commercially available. This AS-OCT system has enabled detailed biometry measurements from the corneal surface to the posterior surface of the lens by elongation of the imaging depth range and improvement of the sensitivity level of performance. ${ }^{23,24}$ In the present study, we attempted to analyze the effects of cycloplegics by using the CASIA2 swept-source AS-OCT system.

\section{Materials and Methods}

\section{Subjects}

Participants of the study included 20 healthy volunteers ( 7 males and 13 females, aged 22-34 years [mean \pm standard deviation: $28.6 \pm 3.5$ years]). All subjects had no history of eye disease, except for refractive errors, and had a bestcorrected visual acuity of 20/20 or better. Exclusion criteria were a history of any previous history of ocular disease, ophthalmic surgery, or laser treatment. Subjects who took systemic medication that might affect accommodation were also excluded.

Each subject's refraction was examined by the ARK-1 autorefractor (NIDEK Co. Ltd., Gamagori, Japan). The dry refraction of the right eye was $-5.3 \pm 3.4 \mathrm{D}$ (mean \pm standard deviation), ranging from -0.25 to $-11.0 \mathrm{D}$, while that of the left eye was $-5.5 \pm 3.4 \mathrm{D}$, ranging from 0.00 to $-11.75 \mathrm{D}$. Thus, there was no statistically significant difference in the refraction of both eyes $(p=0.909$, Mann-Whitney $U$-test). The subject's accommodation was also examined using the ARK-1 autorefractor to ensure the ability to accommodate $5 \mathrm{D}$ or more.

Guidelines outlined by the World Medical Association's Declaration of Helsinki were followed. All subjects received a full explanation of the procedures and provided written informed consent prior to their participation in the study. The study protocol was approved by the institutional review board of Kyorin University School of Medicine (project H30-099).

\section{Methods}

Both eyes of all subjects were examined using the CASIA2 swept-source AS-OCT system. This AS-OCT device has a swept-source laser that operates at a central wavelength of 1,310 $\mathrm{nm}$ and a scan rate of 50,000 A-scans per second. The maximum imaging area is $16.0 \mathrm{~mm} \times$ $16.0 \mathrm{~mm}$ and the maximum imaging depth is $11.0 \mathrm{~mm}$. The simultaneous biometry of all anterior segment structures, including the cornea, anterior chamber, and crystalline lens, can be performed.

All OCT images were obtained in a dim examination room. During measurements, subjects were asked to fixate on the co-axial accommodative target image in the OCT device. The negative or positive lens was set to compensate for the subject's spherical ametropia for nearequivalent spherical refractive correction. Afterward, $-5.0 \mathrm{D}$ lens was added to stimulate the physiologic accommodation using an optical system in the OCT system. The eye was centered by the active eye tracker of the OCT system. All images were collected by two experienced operators (M. Y. and S. S.).

Measurements were conducted with or without cycloplegia. A compounding agent (tropicamide/phenylephrine) consisting of $0.5 \%$ tropicamide and $0.5 \%$ phenylephrine (Mydrin-P ophthalmic solution; Santen Pharmaceutical Co., Osaka, Japan) was instilled into the right eye, while $1 \%$ cyclopentolate (Cyplegin 1\% ophthalmic solution; Santen Pharmaceutical Co.) was instilled into the left eye. These eyedrops were instilled twice with a 5-min interval in between and the OCT images of the eye were measured at $45 \mathrm{~min}$ after the second instillation.

The biometric parameters in this study included central corneal thickness (CCT), pupil diameter (PD), anterior chamber depth (ACD), lens thickness (LT), and horizontal radii of the lens' anterior and posterior surface curvatures (LAC and LPC). The measurement tool provided with the CASIA2 was used to determine these biometric components. The boundaries of the cornea and lens were outlined semi-manually to 
achieve the anterior-segment biometry. The positioning of the anterior and posterior surfaces of the lens on the horizontal meridian was traced; additionally, the radius of the crystalline lens was determined by measurements that permitted circular fitting to the anterior and posterior lens surface.

Prism 7 for Mac OS X (GraphPad Software Inc., La Jolla, CA, USA) was used for statistical analyses. The Wilcoxon signed-rank test or Mann-Whitney $U$-test was used for comparison. P-values of less than 0.05 were considered to be statistically significant.

\section{Results}

Table 1 shows biometric parameters in the relaxed and accommodative states of both eyes with/without cycloplegia. There were no statistically significant differences in any biometric parameters between the right and left eyes in the relaxed state without cycloplegia (Mann-Whitney $U$-test). As shown in Figure 1, ACD, LAC, and LPC decreased and LT increased during 5.0 D accommodative stimulation in both eyes with statistical significance $(\mathrm{P}=0.012$ and 0.020 for PD of right eyes and left eyes; $\mathrm{P}<0.0001$ for ACD, LT, and LAC of both eyes; and P $=0.012$ and 0.042 for LPC of right eyes and left eyes, respectively).

Tropicamide/phenylephrine was instilled in the right eye and cyclopentolate was instilled in the left eye for cycloplegia. Refraction of the right eye was $-5.0 \pm 3.3 \mathrm{D}$ and that of the left eye was $-5.0 \pm 3.3 \mathrm{D}$, respectively, following the instillation of the cycloplegics. The refractions of both eyes become less myopic after cycloplegia, with statistical significance $(\mathrm{P}=0.0068$ for the right eye and $\mathrm{P}<0.0001$ for the left eye, respectively). Biometric parameters in the pre-cycloplegia state and the postcycloplegia state of both eyes are presented in Table 2 and Figure 2. A significant increase in PD was observed after the administration of tropicamide/phenylephrine and cyclopentolate $(\mathrm{P}<0.0001$ for both eyes). $\mathrm{PD}$ of right eyes was significantly larger than that of left eyes $(\mathrm{P}=0.0026)$. There were no changes in $\mathrm{CCT}(\mathrm{P}=0.79$ for the right eye and $\mathrm{P}=0.76$ for the left eye, respectively). Both tropicamide/ phenylephrine and cyclopentolate eyedrops led to outcomes of deeper ACD, thinner LT, and flatter LAC ( $<0.0001$ for ACD and LAC of both eyes, $\mathrm{P}=0.030$ for $\mathrm{LT}$ of right eyes, and $\mathrm{P}<0.0001$ for LT of left eyes, respectively). LPC did not show statistically significant changes after either tropicamide/ phenylephrine or cyclopentolate instillation in this study. When both eyes of ACD, LT, LAC, and LPC with cycloplegia were compared, all parameters were not statistically different ( $\mathrm{P}=0.79, \mathrm{P}=0.92, \mathrm{P}=0.86, \mathrm{P}=0.88$, respectively).

Biometric parameters in the relaxed state (Re.) and accommodative state (Acc.) of both eyes were examined after cycloplegia (Table 1). There were no significant differences in any parameters despite $5.0 \mathrm{D}$ of accommodative stimulation in both eyes.

\section{Discussion}

In the present study, we successfully analyzed the ocular biometric components, including lens parameters, using

Table I Biometric Parameters in the Relaxed State (Relax) and with Accommodative Stimulation (Accom.) of Both Eyes with/Without Cycloplegia

\begin{tabular}{|c|c|c|c|c|c|c|c|c|}
\hline Eye & Cycloplegia & State & PD (mm) & CCT $(\mu \mathrm{m})$ & $\operatorname{ACD}(\mathrm{mm})$ & LT (mm) & LAC (mm) & LPC (mm) \\
\hline \multirow[t]{6}{*}{ Right } & \multirow[t]{3}{*}{ None } & Relax & $4.44 \pm 0.77$ & $527.4 \pm 33.2$ & $3.17 \pm 0.24$ & $3.73 \pm 0.27$ & $11.97 \pm 1.50$ & $5.86 \pm 0.57$ \\
\hline & & Accom. & $4.07 \pm 0.80$ & $528.0 \pm 33.6$ & $3.03 \pm 0.25$ & $3.90 \pm 0.29$ & $9.41 \pm 1.70$ & $5.62 \pm 0.49$ \\
\hline & & $P$ & 0.012 & 0.29 & $\leq 0.0001$ & $\leq 0.000 \mathrm{I}$ & $\leq 0.0001$ & 0.012 \\
\hline & \multirow{3}{*}{$\begin{array}{l}\text { Tropicamide } \\
\text { /phenylephrine }\end{array}$} & Relax & $6.95 \pm 0.45$ & $529.6 \pm 33.9$ & $3.26 \pm 0.24$ & $3.7 \mathrm{I} \pm 0.25$ & $|2.7| \pm 1.72$ & $5.91 \pm 0.48$ \\
\hline & & Accom. & $6.96 \pm 0.43$ & $530.5 \pm 33.9$ & $3.23 \pm 0.24$ & $3.75 \pm 0.26$ & $12.02 \pm 1.77$ & $5.80 \pm 0.49$ \\
\hline & & $P$ & 0.41 & 0.35 & 0.47 & 0.52 & 0.23 & 0.48 \\
\hline \multirow[t]{6}{*}{ Left } & \multirow[t]{3}{*}{ None } & Relax & $4.35 \pm 0.68$ & $526.7 \pm 33.0$ & $3.15 \pm 0.23$ & $3.77 \pm 0.27$ & $11.35 \pm 1.92$ & $5.84 \pm 0.55$ \\
\hline & & Accom. & $4.06 \pm 0.83$ & $526.7 \pm 33.4$ & $3.04 \pm 0.24$ & $3.90 \pm 0.28$ & $9.54 \pm 1.46$ & $5.61 \pm 0.41$ \\
\hline & & $P$ & 0.020 & 0.79 & $\leq 0.000 \mathrm{I}$ & $\leq 0.000 \mathrm{I}$ & $\leq 0.000 \mathrm{I}$ & 0.042 \\
\hline & \multirow[t]{3}{*}{ Cyclopentolate } & Relax & $6.41 \pm 0.63$ & $528.5 \pm 32.9$ & $3.26 \pm 0.22$ & $3.70 \pm 0.24$ & $12.79 \pm 1.75$ & $5.99 \pm 0.60$ \\
\hline & & Accom. & $6.40 \pm 0.69$ & $527.8 \pm 33.9$ & $3.24 \pm 0.27$ & $3.72 \pm 0.24$ & $12.65 \pm 1.84$ & $5.87 \pm 0.57$ \\
\hline & & $P$ & 0.67 & 0.14 & 0.66 & 0.76 & 0.68 & 0.58 \\
\hline
\end{tabular}

Notes: Results are given as means \pm standard deviations. Tropicamide/phenylephrine was instilled in the right eye and cyclopentolate was instilled in the left eye. P-values are calculated by Wilcoxon matched-pairs signed-rank test.

Abbreviations: PD, pupil diameter; CCT, central corneal thickness; ACD, anterior chamber depth; LT, lens thickness; LAC, radius of the lens' anterior surface curvature; LPC, radius of the lens' posterior surface curvature. 

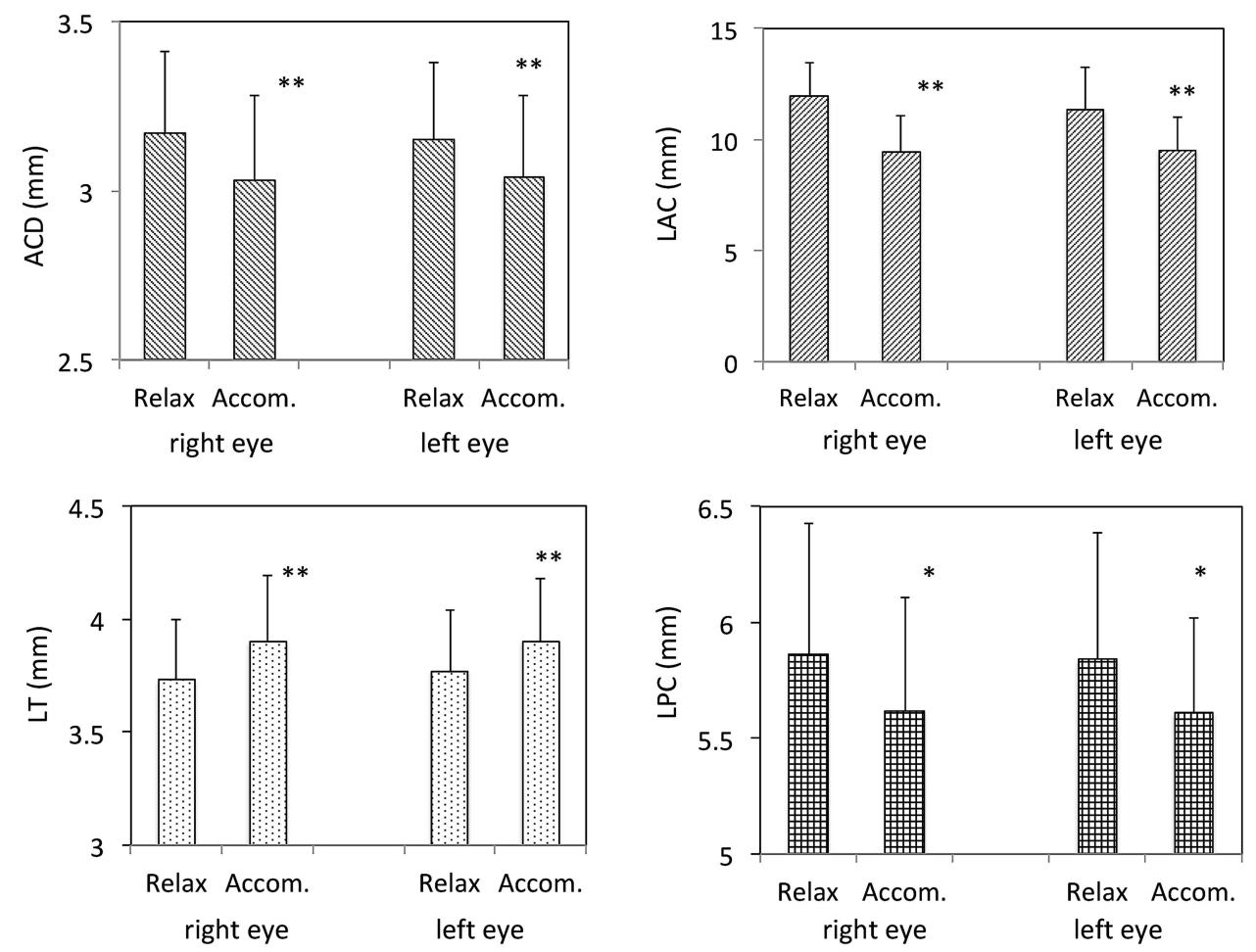

Figure I Biometric parameters in the relaxed state (Relax) and with accommodative stimulation (Accom.) without cycloplegia of both eyes. Anterior chamber depth (ACD), radius of the lens' anterior surface curvature (LAC), and radius of the lens' posterior surface curvature (LPC) decreased and lens thickness (LT) increased during 5.0 $\mathrm{D}$ accommodative stimulation in both eyes with statistical significance $(* \mathrm{P}<0.05, * * \mathrm{P}<0.000 \mathrm{I}$, respectively).

a commercially available AS-OCT system (CASIA2). The AS-OCT system used in the current study was capable of simultaneous visualization of all optical components of the anterior segment in a single frame. ${ }^{22,23}$ Biometric components such as LT, LAC, and LPC in our study appeared comparable to findings of such in previous studies. ${ }^{17-23}$ The results of our study during accommodation are also in accordance with previous reports of biometric analysis. ${ }^{17-23}$ The biometric analysis showed that the LT increased, both LAC and LPC decreased, and the ACD decreased with accommodation, which suggested the occurrence of both steepening and anterior movement of the lens. Although both the anterior and posterior surfaces of the lens became steeper, the steepening of the anterior lens curvature was greater than that of the posterior curvature.

With the application of cycloplegics, the LT decreased resulting in an essentially equivalent increase in ACD. A flattening of the lens anterior surface was observed, but the change in the lens posterior surface was not significant in this study. Refractive changes after cycloplegia found in this study appear to reflect the changes in these biometric parameters of the lens. No significant alterations were observed in

Table 2 Biometric Parameters in the Precyclopegia State (Pre.) and Postcycloplegia State (Post.) of Both Eyes in the Relaxed State

\begin{tabular}{|l|l|l|l|l|l|l|l|}
\hline Eye & Cycloplegia & PD $(\mathbf{m m})$ & CCT $(\boldsymbol{\mu m})$ & ACD $(\mathbf{m m})$ & LT $(\mathbf{m m})$ & LAC $(\mathbf{m m})$ & LPC $(\mathbf{m m})$ \\
\hline Right & Pre. & $4.44 \pm 0.77$ & $527.4 \pm 33.2$ & $3.17 \pm 0.24$ & $3.73 \pm 0.27$ & $11.97 \pm 1.50$ & $5.86 \pm 0.57$ \\
& Post. & $6.95 \pm 0.45$ & $529.6 \pm 33.9$ & $3.26 \pm 0.24$ & $3.71 \pm 0.25$ & $12.71 \pm 1.72$ & $5.91 \pm 0.48$ \\
& P & $\leq 0.0001$ & 0.79 & $\leq 0.0001$ & 0.030 & $\leq 0.0001$ & 0.98 \\
\hline \multirow{2}{*}{ Left } & Pre. & $4.35 \pm 0.68$ & $526.7 \pm 33.0$ & $3.15 \pm 0.23$ & $3.77 \pm 0.27$ & $11.35 \pm 1.92$ & $5.84 \pm 0.55$ \\
& Post. & $6.41 \pm 0.63$ & $528.5 \pm 32.9$ & $3.26 \pm 0.22$ & $3.70 \pm 0.24$ & $12.79 \pm 1.75$ & $5.99 \pm 0.60$ \\
& P & $\leq 0.0001$ & 0.76 & $\leq 0.0001$ & $\leq 0.0001$ & $\leq 0.0001$ & 0.37 \\
\hline
\end{tabular}

Notes: Tropicamide/phenylephrine was instilled in the right eye and cyclopentolate was instilled in the left eye. Results are given as means \pm standard deviations. P-values are calculated by Wilcoxon matched-pairs signed-rank test.

Abbreviations: PD, pupil diameter; CCT, central corneal thickness; ACD, anterior chamber depth; LT, lens thickness; LAC, radius of the lens' anterior surface curvature; and LPC, radius of the lens' posterior surface curvature. 

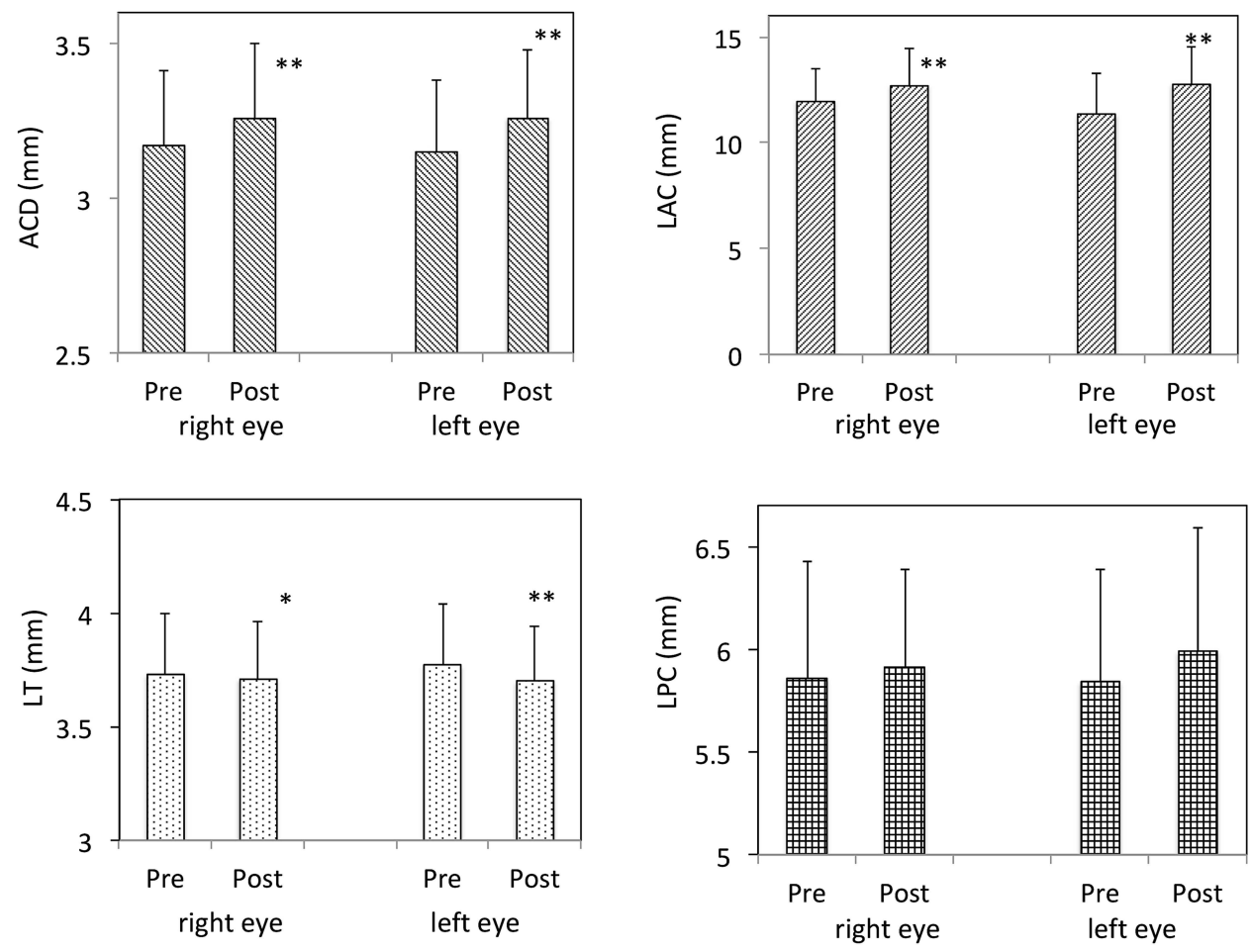

Figure 2 Biometric parameters in the pre-cycloplegia state (Pre.) and postcycloplegia state (Post.) of both eyes. Tropicamide/phenylephrine was instilled in the right eye and cyclopentolate was instilled in the left eye for cycloplegia. Both tropicamide/phenylephrine and cyclopentolate eyedrops led to outcomes of deeper anterior chamber depth (ACD), thinner lens thickness (LT), and flatter radius of the lens' anterior surface curvature (LAC) ( $* \mathrm{P}<0.05$, **P $<0.000$ I, respectively). Radius of the lens' posterior surface curvature (LPC) did not show statistically significant changes.

any biometric parameters with $-5.0 \mathrm{D}$ accommodative stimulation in both eyes after cycloplegia. Our results suggest that the cycloplegic effects of tropicamide/phenylephrine are comparable to those of cyclopentolate, although subjects' profiles should be taken into consideration. ${ }^{4,7-10}$ Our study only included young adults with myopia. Cyclopentolate is reported to produce a significant refractive change that is more positive than that of tropicamide in children and in subjects with hyperopic refractive errors. ${ }^{4}$ Similar measurements among subjects of younger ages or different refractive statuses might yield different results.

Further, there are several limitations in our study. First, as in most other studies using AS-OCT, the whole lens shape cannot be analyzed through the pupil. ${ }^{17-24}$ The curvature radius obtained by fitting the circular curves may not exactly express the shape of the lens because the variability of the measurements and the asphericity of the lens. ${ }^{25}$ Second, we tested a compounded agent consisting of tropicamide and phenylephrine in this study because it is frequently used for mydriasis and cycloplegia in clinical settings in Japan. We assume that the cycloplegic effects of this compounded agent are mostly derived from tropicamide because previous reports showed that phenylephrine has no effect on the accommodative system performed by objective measurement methods, including AS-OCT. ${ }^{21,26,27}$ However, it is impossible to fully distinguish the effects of tropicamide and phenylephrine individually in the present research. Third, we measured the biometric components 45 min after administration. According to the literature, the cycloplegic effect of cyclopentolate begins at 25-75 $\mathrm{min}$ after the administration of the drop and recovery occurs through 6-24 $\mathrm{h}$ later. ${ }^{4}$ Tropicamide is characterized by a rapid onset of action; its cycloplegic effect appears 20-30 min after administration and recovery occurs $6 \mathrm{~h}$ later. We chose the time point of 45 minutes to compare the pharmacological actions of both cycloplegics. Measurements at multiple time points may support a better understanding of cycloplegics.

In conclusion, we successfully analyzed the ocular biometric components, including lens parameters, using a commercially available AS-OCT system. After the topical administration of cycloplegic eyedrops, changes in ACD, ACL, and LT extinguished regardless of accommodative stimuli. Our results suggest that both tropicamide/ phenylephrine and cyclopentolate eyedrops have enough cycloplegic effects in young adults. 


\section{Author Contributions}

All authors made substantial contributions to conception and design, acquisition of data, or analysis and interpretation of data; took part in drafting the article or revising it critically for important intellectual content; gave final approval of the version to be published; and agree to be accountable for all aspects of the work.

\section{Funding}

This study was supported by a grant (19FA1010) from the Ministry of Health, Labor and Welfare, Japan.

\section{Disclosure}

The authors have no competing interests, including relevant financial interests, activities, and affiliations.

\section{References}

1. Charman WN. The eye in focus: accommodation and presbyopia. Clin Exp Optom. 2008;91:207-225. doi:10.1111/j.1444-0938.2008.00256.x

2. Koretz JF, Cook CA, Kaufman PL. Accommodation and presbyopia in the human eye; changes in the anterior segment and crystalline lens with focus. Invest Ophthalmol Vis Sci. 1997;38:569-578.

3. Zhao J, Mao J, Luo R, Li F, Pokharel GP, Ellwein LB. Accuracy of noncycloplegic autorefraction in school-age children in China. Optom Vis Sci. 2004;81:49-55. doi:10.1097/00006324-200401000-00010

4. Yazdani N, Sadeghi R, Momeni-Moghaddam H, Zarifmahmoudi L, Ehsaei A. Comparison of cyclopentolate versus tropicamide cycloplegia: a systematic review and meta-analysis. $J$ Optom. 2018;11:135-143. doi:10.1016/j.optom.2017.09.001

5. Kawamoto K, Hayasaka S. Cycloplegic refractions in Japanese children: a comparison of atropine and cyclopentolate. Ophthalmologica. 1997;211:57-60. doi:10.1159/000310758

6. Wakayama A, Nishina S, Miki A, Utsumi T, Sugasawa J, Hayashi T. Incidence of side effects of topical atropine sulfate and cyclopentolate hydrochloride for cycloplegia in Japanese children: a multicenter study. Jpn J Ophthalmol. 2018;62:531-536. doi:10.1007/s10384018-0612-7

7. Egashira SM, Kish LL, Twelker JD, Mutti DO, Zadnik K, Adams AJ. Comparison of cyclopentolate versus tropicamide cycloplegia in children. Optom Vis Sci. 1993;70:1019-1026. doi:10.1097/ 00006324-199312000-00005

8. Lin LL, Shih YF, Hsiao CH, Su TC, Chen CJ, Hung PT. The cycloplegic effects of cyclopentolate and tropicamide on myopic children. J Ocul Pharmacol Ther. 1998;14:331-335. doi:10.1089/ jop.1998.14.331

9. Hofmeister EM, Kaupp SE, Schallhorn SC. Comparison of tropicamide and cyclopentolate for cycloplegic refractions in myopic adult refractive surgery patients. $J$ Cataract Refract Surg. 2005;31:694-700. doi:10.1016/j.jcrs.2004.10.068

10. Yoo SG, Cho MJ, Kim US, Baek SH. Cycloplegic refraction in hyperopic children: effectiveness of a $0.5 \%$ tropicamide and $0.5 \%$ phenylephrine addition to $1 \%$ cyclopentolate regimen. Korean J Ophthalmol. 2017;31:249-256. doi:10.3341/kjo.2016.0007

11. Sheppard AL, Evans CJ, Singh KD, Wolffsohn JS, Dunne MC, Davies LN. Three-dimensional magnetic resonance imaging of the phakic crystalline lens during accommodation. Invest Ophthalmol Vis Sci. 2011;52:3689-3697. doi:10.1167/iovs.10-6805
12. Kasthurirangan S, Markwell EL, Atchison DA, Pope JM. MRI study of the changes in crystalline lens shape with accommodation and aging in humans. J Vis. 2011;11:19. doi:10.1167/11.3.19

13. Palamar M, Alkan Z, Egrilmez S, Yagci A. Influences of tropicamide on anterior segment parameters with pentacam in healthy individuals. J Ocul Pharmacol Ther. 2013;29:349-352. doi:10.1089/jop.2012. 0145

14. Palamar M, Egrilmez S, Uretmen O, Yagci A, Kose S. Influences of cyclopentolate hydrochloride on anterior segment parameters with pentacam in children. Acta Ophthalmol. 2011;89:e461-5. doi:10.11 11/j.1755-3768.2011.02122.x

15. Yasuno Y, Madjarova VD, Makita S, et al. Three-dimensional and high-speed swept-source optical coherence tomography for in vivo investigation of human anterior eye segments. Opt Express. 2005;13:10652-10664. doi:10.1364/OPEX.13.010652

16. Gora M, Karnowski K, Szkulmowski M, et al. Ultra high-speed swept source OCT imaging of the anterior segment of human eye at $200 \mathrm{kHz}$ with adjustable imaging range. Opt Express. 2009;17:14880-14894. doi:10.1364/OE.17.014880

17. Du C, Shen M, Li M, Zhu D, Wang MR, Wang J. Anterior segment biometry during accommodation imaged with ultralong scan depth optical coherence tomography. Ophthalmology. 2012;119:2479-2485. doi:10.1016/j.ophtha.2012.06.041

18. Gambra E, Ortiz S, Perez-Merino P, Gora M, Wojtkowski M, Marcos S. Static and dynamic crystalline lens accommodation evaluated using quantitative 3-D OCT. Biomed Opt Express. 2013;4:1595-1609. doi:10.1364/BOE.4.001595

19. Zhong J, Tao A, Xu Z, Zhu D, Wang MR, Wang J. Whole eye axial biometry during accommodation using ultra-long scan depth optical coherence tomography. Am J Ophthalmol. 2014;157:1064-1069. doi:10.1016/j.ajo.2014.01.016

20. Sun Y, Fan S, Zheng H, Dai C, Ren Q, Zhou C. Noninvasive imaging and measurement of accommodation using dual channel SD-OCT. Curr Eye Res. 2014;39:611-619. doi:10.3109/02713683.2013.860991

21. Zhang J, Ni Y, Li P, et al. Anterior segment biometry with phenylephrine and tropicamide during accommodation imaged with ultralong scan depth optical coherence tomography. J Ophthalmol. 2019;6827215.

22. Neri A, Ruggeri M, Protti A, Leaci R, Gandolfi SA, Macaluso C. Dynamic imaging of accommodation by swept-source anterior segment optical coherence tomography. $J$ Cataract Refract Surg. 2015;41:501-510. doi:10.1016/j.jcrs.2014.09.034

23. Shoji T, Kato N, Ishikawa S, et al. In vivo crystalline lens measurements with novel swept-source optical coherent tomography: an investigation on variability of measurement. BMJ Open Ophthalmol. 2017;1:e000058. doi:10.1136/bmjophth-2016-000058

24. Shoji T, Kato N, Ishikawa S, et al. Association between axial length and in vivo human crystalline lens biometry during accommodation: a swept-source optical coherence tomography study. Jpn J Ophthalmol. 2020;64:93-101. doi:10.1007/s10384-019-00700-8

25. Martínez-Enríquez E, Sun M, Velasco-Ocana M, Birkenfeld J, PérezMerino P, Marcos S. Optical coherence tomography based estimates of crystalline lens volume, equatorial diameter, and plane position. Invest Ophthalmol Vis Sci. 2016;57:OCT600-10. doi:10.1167/ iovs.15-18933

26. Richdale K, Bailey MD, Sinnott LT, Kao CY, Zadnik K, Bullimore MA. The effect of phenylephrine on the ciliary muscle and accommodation. Optom Vis Sci. 2012;89:1507-1511. doi:10.10 97/OPX.0b013e318269c8d0

27. Del Águila-carrasco AJ, Lara F, Bernal-Molina P, et al. Effect of phenylephrine on static and dynamic accommodation. J Optom. 2019;12:30-37. doi:10.1016/j.optom.2018.01.005 


\section{Publish your work in this journal}

Clinical Ophthalmology is an international, peer-reviewed journal covering all subspecialties within ophthalmology. Key topics include Optometry; Visual science; Pharmacology and drug therapy in eye diseases; Basic Sciences; Primary and Secondary eye care; Patient Safety and Quality of Care Improvements. This journal is indexed on PubMed
Central and CAS, and is the official journal of The Society of Clinical Ophthalmology (SCO). The manuscript management system is completely online and includes a very quick and fair peer-review system, which is all easy to use. Visit http://www.dovepress.com/ testimonials.php to read real quotes from published authors. 